\title{
Article \\ Osteopontin Serum Concentration and Metabolic Syndrome in Male Psoriatic Patients
}

\author{
Joanna Bartosińska 1,*D , Joanna Przepiórka-Kosińska ${ }^{2} \mathbb{D}$, Beata Sarecka-Hujar ${ }^{3} \mathbb{D}$, Dorota Raczkiewicz ${ }^{4} \mathbb{D}_{\text {, }}$ \\ Małgorzata Kowal ${ }^{2}$, Katarzyna Chyl-Surdacka ${ }^{2}$, Jarosław Bartosiński ${ }^{5}$, Jakub Kosiński ${ }^{6}$, Dorota Krasowska ${ }^{2} \mathbb{D}$ \\ and Grażyna Chodorowska ${ }^{2}$
}

Citation: Bartosińska, J.; Przepiórka-Kosińska, J.; Sarecka-Hujar, B.; Raczkiewicz, D.; Kowal, M.; Chyl-Surdacka, K.; Bartosiński, J.; Kosiński, J.;

Krasowska, D.; Chodorowska, G. Osteopontin Serum Concentration and Metabolic Syndrome in Male Psoriatic Patients. J. Clin. Med. 2021, 10, 755. https://doi.org/10.3390 /jcm10040755

Academic Editor: Keiichi Yamanaka

Received: 14 December 2020

Accepted: 10 February 2021

Published: 13 February 2021

Publisher's Note: MDPI stays neutral with regard to jurisdictional claims in published maps and institutional affiliations.

Copyright: (C) 2021 by the authors. Licensee MDPI, Basel, Switzerland. This article is an open access article distributed under the terms and conditions of the Creative Commons Attribution (CC BY) license (https:/ / creativecommons.org/licenses/by/ $4.0 /)$.
1 Department of Cosmetology and Aestetic Medicine, Medical University of Lublin, ul. Chodźki 1, 20-093 Lublin, Poland

2 Department of Dermatology, Venereology and Pediatric Dermatology, Medical University of Lublin, ul. Staszica 11, 20-081 Lublin, Poland; asia880124@wp.pl (J.P.-K.); kowalma71@o2.pl (M.K.); kasiachyl@gmail.com (K.C.-S.); dorota.krasowska@umlub.pl (D.K.); grazyna.chodorowska@umlub.pl (G.C.)

3 Department of Basic Biomedical Science, Faculty of Pharmaceutical Sciences in Sosnowiec, Medical University of Silesia in Katowice, ul. Kasztanowa 3, 41-200 Sosnowiec, Poland; bsarecka-hujar@sum.edu.pl

4 Department of Medical Statistics, School of Public Health, Center of Postgraduate Medical Education, ul. Kleczewska 61/63, 01-826 Warsaw, Poland; dorota.bartosinska@gmail.com

5 Department of Anaesthesiology and Intensive Therapy, Independent Public Clinical Hospital No. 4 in Lublin, ul. Jaczewskiego 8, 20-954 Lublin, Poland; jeremybartosinski@gmail.com

6 Department of Rehabilitation and Orthopedics, Medical University of Lublin, ul. Jaczewskiego 8, 20-954 Lublin, Poland; kuba.kosinski@gmail.com

* Correspondence: jbartosinski@gmail.com; Tel.: +48-602-724-298

\begin{abstract}
Psoriasis (Ps) is an immune-mediated inflammatory skin disease that is widely associated with the clinical features of metabolic syndrome (MetS), including hypertension, abdominal obesity, insulin resistance, type 2 diabetes and dyslipidemia. Osteopontin (OPN), a multifunctional protein involved in the modulation of inflammatory processes, may contribute to the development of atherosclerosis and MetS. Therefore, the aim of the study was the assessment of the correlation between OPN concentration in the peripheral blood and the presence of MetS as well as its particular components in the Ps patients. The study comprised 107 male Ps patients (50 patients with MetS and 57 without MetS) and 38 healthy volunteers (HVs). The concentration of OPN in serum was determined using enzyme-linked immunosorbent assay (ELISA) method. Fasting blood glucose and lipid profile components: total cholesterol (total CHOL), high-density lipoprotein cholesterol (HDL-CHOL), low-density lipoprotein cholesterol (LDL-CHOL), triglycerides (TG) were examined. Ps patients with MetS had significantly higher obesity, systolic blood pressure, TG, CHOL/HDL, LDL/HDL and TG/HDL ratios than Ps patients without MetS. OPN serum concentration was significantly higher in the Ps patients than in the HVs $(p=0.022)$ but not significantly different between the Ps patients with and without MetS $(p=0.275)$. OPN serum concentration in Ps patients correlated negatively with total CHOL $(p=0.004)$ and TG $(p=0.009)$. OPN is increased in Ps patients and may serve as a biomarker of some lipid abnormalities in them.
\end{abstract}

Keywords: psoriasis; metabolic syndrome; osteopontin

\section{Introduction}

Psoriasis (Ps) is a chronic inflammatory skin disease of complex pathogenesis with genetic predisposition, environmental factors and immunological disturbances. Chronic inflammation, which is the essence of pathophysiological phenomena in Ps, is also a factor predisposing these patients to the occurrence of systemic disorders, including diabetes, hypertension, lipid disorders and cardiovascular diseases (CVDs). Numerous inflammatory mediators produced by various cells, including Th-Th1, Th17, Th22 subpopulations, which produce such cytokines as tumor necrosis factor $\alpha$ (TNF- $\alpha$ ), interleukin 17 (IL-17), 
IL-22, participate in the formation of psoriatic plaques as well as in the development of atherosclerosis and CVDs [1,2].

The increased risk of hypertension, abdominal obesity, insulin resistance and dyslipidemia in Ps patients contributes to the development of metabolic syndrome (MetS) [1,3,4]. The probability of obesity in Ps patients is almost twice as high as in the general population [3,4]. Moreover, it has been demonstrated that with the increase in body mass index (BMI), the risk of Ps increases [4], and the increased production of TNF- $\alpha$ in obese patients shows a positive correlation with the amount of IL-17 produced [5]. Both obesity and systemic inflammation contribute to the development of insulin resistance and type 2 diabetes [4]. Patients with Ps have an increased risk of dyslipidemia, i.e., elevated triglycerides, LDL (low-density lipoprotein) cholesterol, VLDL (very-low-density lipoprotein) and decreased HDL (high-density lipoprotein) cholesterol [2-4].

The results of many studies indicate that an increased risk of myocardial infarction may be observed in Ps patients who do not have traditional risk factors [1-4]. It is suggested that in Ps patients a faster development of atherosclerosis is observed [5]. The occurrence of other CVDs that may be associated with Ps, including atrial fibrillation, additionally increases the risk of ischemic stroke, coronary heart disease and sudden cardiovascular death [4]. Similar to myocardial infarction, the risk of stroke is particularly high in patients with severe Ps and is independent of other known risk factors for stroke [6,7].

The previous data indicated that osteopontin (OPN) participated in both acute and chronic inflammatory processes [8]. At the inflammatory site, OPN is secreted mainly by $\mathrm{T}$ lymphocytes and activated macrophages, whereas circulating monocytes do not have this property. OPN also stimulates the chemotaxis of macrophages and the secretion of IL-12 as well as promotes the chemotaxis and the adhesion of T lymphocytes and their proliferation [8-14]. It has been found that low concentration values or the lack of expression of OPN leads to the reduction of inflammation [15]. OPN also contributes to the formation of atherosclerotic plaque $[11,14,16]$. In damaged blood vessels, OPN takes part in their repair and remodeling by increasing proliferation and migration of smooth muscle cells and endothelium $[10,11,14]$. OPN is also an important regulator of calcification and vascular mineralization [11,14]. It was found that the increased concentration of OPN was associated with a higher risk of developing CVDs, regardless of the presence of traditional risk factors [11]. Increased plasma OPN levels in patients with coronary artery disease and chronic heart failure suggest that it may be a new marker of CVDs [14]. OPN is believed to be involved in the development of obesity, insulin resistance and type 2 diabetes $[10,17,18]$. In the course of diabetes, OPN takes part in the development of changes in blood vessels by influencing the nuclear factor of activated T cells. Both obesity and type 2 diabetes coexist with non-alcoholic fatty liver disease, which may be related to the observed increase in OPN levels in hepatocytes [1,2,17].

The participation of OPN, a pro-inflammatory, angiogenic and anti-apoptotic protein, may be significant in Ps pathogenesis, contributing to the occurrence of chronic inflammation and thus to the development of coexisting diseases, such as obesity, diabetes, insulin resistance, metabolic disorders, atherosclerosis and other CVDs.

Therefore, the aim of the study was the assessment of the correlation between OPN concentration in the peripheral blood and the presence of MetS as well as its particular components.

\section{Materials and Methods}

\subsection{The Study Groups}

The study comprised 107 male Ps patients and 38 age-matched healthy male volunteers (HVs). The Ps patients and HVs underwent physical examination, including the measurement of body weight, height, waist and hip circumference, arterial pressure and heart rate. Body mass index (BMI) and waist-to-hip ratio (WHR) were calculated in Ps patients and HVs. 
The exclusion criteria were female sex, treatment with topical and/or systemic antipsoriatic medications less than 3 months before the recruitment into the study, history of malignancy and anti-cancer treatment.

The inclusion criteria were male sex, psoriasis diagnosis confirmed by dermatologist.

Female psoriatic patients were excluded due to the possible influence of sexual hormones, i.e., estrogens on OPN gene [19]. Moreover, there are some observations that OPN levels differ in pre- and postmenopausal women and may be associated with bone loss and osteoporosis [20,21].

Informed consent was obtained from each individual. The study protocol complies with the ethical guidelines of the 1975 Declaration of Helsinki. The study was approved by the local ethics committee (KE-0254/30/2016).

\subsection{Characteristics of Psoriasis in the Studied Ps Patients}

Medical history, including the duration of Ps, family history and co-morbidities was taken. The severity of Ps was assessed using Psoriasis Area and Severity Index (PASI), Body Surface Area (BSA) and Physician Global Assessment (PGA).

The Ps patients who had not received any topical and systemic anti-psoriatic treatment for at least 3 months before the recruitment were included in the study.

The age of Ps onset ranged from less than 1 year to 65 years, with an average of 25 years. Psoriasis duration was from a few months to 48 years, 19 years on average. In the studied patients, the severity of Ps skin lesions expressed by PASI was between 3 and 45 with an average of 13 . The extent of Ps skin lesions expressed by BSA in the studied patients was from $5 \%$ to $90 \%, 25 \%$ on average. PGA was minimal in 13 patients $(12.15 \%)$, mild in 57 patients (55.74\%), moderate in another 35 individuals (32.71\%) and severe in 2 patients $(1.87 \%)$.

\subsection{Assessment of Serum OPN Concentration in Ps Patients and HVs}

In Ps patients and HVs, peripheral blood samples were collected. The blood samples were centrifuged for $15 \mathrm{~min}$ at $1000 \times g$ and stored at $-80^{\circ} \mathrm{C}$ until tested. The concentration of OPN in serum was determined using enzyme-linked immunosorbent assay (ELISA) kit (Quantikine ${ }^{\circledR}$ ELISA Human Osteopontin, R\&D Systems, Inc., Minneapolis, MN, USA).

\subsection{Determining the Presence of MetS in Ps Patients and HVs}

In the analyzed groups, the following laboratory tests were performed: fasting blood glucose and lipid profile components: total cholesterol (total CHOL), HDL cholesterol (HDL-CHOL), LDL cholesterol (LDL-CHOL), triglycerides (TG). The presence of MetS was defined as the coexistence of at least 3 out of the 5 following risk factors:

1. Waist circumference $\geq 94 \mathrm{~cm}$,

2. $\mathrm{TG} \geq 150 \mathrm{mg} / \mathrm{dL}$ or treatment for dyslipidemia,

3. HDL-CHOL $<40 \mathrm{mg} / \mathrm{dL}$ or treatment for dyslipidemia,

4. Systolic blood pressure $\geq 130 \mathrm{mmHg}$ and/or diastolic blood pressure $\geq 85 \mathrm{mmHg}$ or antihypertensive therapy,

5. Fasting glucose $\geq 100 \mathrm{mg} / \mathrm{dL}$ or hypoglycemic treatment.

MetS was found in 50 Ps patients, i.e., 46.73\%, while it was not present in HVs. In the group of Ps patients with MetS, 11 cases (22\%) were treated for dyslipidemia with the use of statins.

\subsection{Statistical Methods}

The data were statistically analyzed using STATISTICA 13 software. The minimum and maximum values and the median and interquartile range (25-75\%) were estimated for continuous variables, as well as absolute numbers $(n)$ and percentages $(\%)$ of the occurrence of items for categorical variables.

The following statistical tests were used: 
- $\quad$ Pearson's chi-square test to compare categorical variables between psoriatic patients and healthy volunteers, between psoriatic patients with and without metabolic syndrome;

- Mann-Whitney's U test to compare: continuous variables between two groups, OPN between two categories of categorical variables;

- Kruskal-Wallis's H test to compare continuous variables between three groups, OPN between more than two categories of categorical variables;

- Spearman's correlation coefficient to correlate OPN with continuous variables.

- $\quad$ The significance level was assumed as 0.05 .

\section{Results}

3.1. Characteristics of Ps Patients and HVs

The studied Ps patients' ages ranged from 18 to 77 years, 47 years on average. The average age of the HV group was 48 years and did not significantly differ from the age of Ps patients $(p=0.953)$.

Overweight and obesity were significantly more common in Ps patients $(35.51 \%$ and $34.58 \%$, respectively) in comparison to $\mathrm{HVs}(15.79 \%$ and $0.00 \%$, respectively), $p<0.001$. WHR of at least 1 was found in $41.12 \%$ of Ps patients, whereas in all HVs WHR was within normal limits. Systolic and diastolic blood pressure, total CHOL and TG serum concentrations, $\mathrm{CHOL} / \mathrm{HDL}$, and TG/HDL ratios were significantly higher in Ps patients than in HVs $(p<0.05)$. However, LDL-CHOL and glucose levels did not differ significantly between Ps patients and HVs ( $p=0.291$ and $p=0.817$, respectively) (Table 1$)$.

\subsection{Comparison of Ps Patients with and without MetS}

The characteristics presented in Table 1 differed significantly between Ps patients with MetS vs. Ps without MetS vs. HVs, except for LDL-CHOL and glucose serum concentration. Ps patients with MetS had significantly higher BMI, WHR, waist circumference, systolic blood pressure, TG, CHOL/HDL, LDL/HDL and TG/HDL ratios than Ps patients without MetS. However, diastolic blood pressure, total CHOL and LDL-CHOL did not differ significantly between Ps patients with and without MetS.

3.3. Comparison of OPN between Ps Patients and HVs as Well as between Ps Patients with and without MetS

OPN serum concentration was significantly higher in the Ps patients than in the HVs $(p=0.022)$ but not significantly different between the Ps patients with and without MetS $(p=0.275)$ (Figure 1).

\subsection{Correlations between OPN and Ps Patients' Characteristics}

OPN serum concentration did not correlate with BMI, WHR, waist circumference, systolic and diastolic blood pressure, glucose, LDL-CHOL and HDL-CHOL concentrations as well as CHOL/HDL and LDL/HDL ratios in Ps patients (Table 2). 
Table 1. Characteristics of the psoriatic patients and the healthy volunteers.

\begin{tabular}{|c|c|c|c|c|c|c|c|c|c|c|c|c|}
\hline Characteristics & Variable & IU & Parameter & $\begin{array}{c}\text { Ps Total } \\
(N=107)\end{array}$ & $\begin{array}{c}\text { HVs } \\
(N=38)\end{array}$ & $\begin{array}{c}p^{\mathrm{a}} \\
\text { Ps Total vs. } \\
\text { HVs }\end{array}$ & $\begin{array}{l}\text { Ps MetS }(+) \\
(N=50)\end{array}$ & $\begin{array}{l}\text { Ps MetS (-) } \\
\quad(N=57)\end{array}$ & $\begin{array}{c}p^{\mathrm{b}} \\
\text { Ps MetS (+) } \\
\text { vs. Ps MetS } \\
\text { (-) vs. HVs }\end{array}$ & $\begin{array}{c}p^{\mathrm{a}} \\
\text { Ps MetS (+) } \\
\text { vs. Ps MetS } \\
(-)\end{array}$ & $\begin{array}{c}p^{\mathrm{a}} \\
\text { Ps MetS (+) } \\
\text { vs. HVs }\end{array}$ & $\begin{array}{c}p^{\mathrm{a}} \\
\text { Ps MetS (-) } \\
\text { vs. HVs }\end{array}$ \\
\hline Age & years & & Me (IQR) & $47(37-58)$ & $48(45-50)$ & 0.953 & $53(43-59)$ & $42(35-57)$ & 0.007 & 0.006 & 0.044 & 0.055 \\
\hline \multirow{8}{*}{ Obesity } & \multirow{4}{*}{ BMI } & $\mathrm{kg} / \mathrm{m}^{2}$ & Me (IQR) & $\begin{array}{c}27.74 \\
(24.54-30-78)\end{array}$ & $\begin{array}{c}22.95 \\
(21.77-24.37) \\
\end{array}$ & $<0.001$ & $\begin{array}{c}29.98 \\
(26.87-32.66) \\
\end{array}$ & $\begin{array}{c}25.76 \\
(23.14-28.07)\end{array}$ & $<0.001$ & $<0.001$ & $<0.001$ & $<0.001$ \\
\hline & & normal & $n(\%)$ & $32(29.91)$ & $32(84.21)$ & \multirow{3}{*}{$<0.001$} & $5(10.00)$ & $27(47.37)$ & \multirow{3}{*}{$<0.001$} & \multirow{3}{*}{$<0.001$} & \multirow{3}{*}{$<0.001$} & \multirow{3}{*}{$<0.001$} \\
\hline & & overweight & $n(\%)$ & 38 (35.51) & $6(15.79)$ & & $20(40.00)$ & $18(31.58)$ & & & & \\
\hline & & obese & $n(\%)$ & 37 (34.58) & $0(0.00)$ & & $25(50.00)$ & $12(21.05)$ & & & & \\
\hline & \multirow{2}{*}{ WHR } & ratio & Me (IQR) & $\begin{array}{c}0.97 \\
(0.94-1.03)\end{array}$ & $\begin{array}{c}0.91 \\
(0.86-0.93)\end{array}$ & $<0.001$ & $\begin{array}{c}1.00 \\
(0.96-1.05)\end{array}$ & $\begin{array}{c}0.96 \\
(0.93-1.00)\end{array}$ & $<0.001$ & 0.001 & $<0.001$ & $<0.001$ \\
\hline & & $\geq 1.0$ & $n(\%)$ & $44(41.12)$ & $0(0.00)$ & $<0.001$ & $28(56)$ & $16(28.07)$ & $<0.001$ & 0.003 & $<0.001$ & $<0.001$ \\
\hline & \multirow{2}{*}{$\begin{array}{c}\text { Waist } \\
\text { circumference }\end{array}$} & $\mathrm{cm}$ & Me (IQR) & $98(90-109)$ & $92(85-94)$ & $<0.001$ & 105 (98-114) & $92(87-103)$ & $<0.001$ & $<0.001$ & $<0.001$ & 0.095 \\
\hline & & $\geq 94 \mathrm{~cm}$ & $n(\%)$ & $69(64.49)$ & $10(26.32)$ & $<0.001$ & $43(86.00)$ & $26(45.61)$ & $<0.001$ & $<0.001$ & $<0.001$ & 0.092 \\
\hline \multirow{3}{*}{$\begin{array}{l}\text { Blood } \\
\text { pressure }\end{array}$} & \multirow{2}{*}{ Systolic } & $\mathrm{mm} \mathrm{Hg}$ & $\mathrm{Me}(\mathrm{IQR})$ & $144(130-152)$ & $130(125-130)$ & $<0.001$ & $146(137-157)$ & 139 (124-146) & $<0.001$ & 0.004 & $<0.001$ & 0.006 \\
\hline & & $\geq 130$ & $n(\%)$ & $82(76.64)$ & $23(60.53)$ & 0.056 & $43(86.00)$ & $39(68.42)$ & 0.016 & 0.032 & 0.006 & 0.567 \\
\hline & Diastolic & $\geq 85$ & $n(\%)$ & $58(54.21)$ & $8(21.05)$ & $<0.001$ & $30(60.00)$ & $28(49.12)$ & 0.001 & 0.260 & 0.001 & 0.011 \\
\hline \multirow{8}{*}{ Lipids } & \multirow{2}{*}{ Total CHOL } & $\mathrm{mg} / \mathrm{dL}$ & $\mathrm{Me}(25-75 \%)$ & 183 (154-208) & 163 (136-185) & 0.003 & 189 (164-217) & 180 (151-203) & 0.005 & 0.166 & 0.001 & 0.044 \\
\hline & & $>190$ & $n(\%)$ & $45(42.06)$ & $6(15.79)$ & 0.004 & $24(48.00)$ & $21(36.84)$ & 0.005 & 0.243 & 0.003 & 0.046 \\
\hline & \multirow{2}{*}{ LDL-CHOL } & $\mathrm{mg} / \mathrm{dL}$ & $\mathrm{Me}(\mathrm{IQR})$ & $99(72-129)$ & $105(92-107)$ & 0.668 & 99 (83-139) & $93(67-126)$ & 0.403 & 0.159 & 0.963 & 0.441 \\
\hline & & $\geq 115$ & $n(\%)$ & 35 (32.71) & $4(10.53)$ & 0.008 & $18(36.00)$ & $17(29.82)$ & 0.015 & 0.497 & 0.005 & 0.049 \\
\hline & \multirow{2}{*}{ HDL-CHOL } & $\mathrm{mg} / \mathrm{dL}$ & $\mathrm{Me}(\mathrm{IQR})$ & $47(38-60)$ & $52(44-59)$ & 0.291 & $39(33-49)$ & $57(46-71)$ & $<0.001$ & $<0.001$ & $<0.001$ & 0.111 \\
\hline & & $\leq 40$ & $n(\%)$ & $30(28.04)$ & $6(15.79)$ & 0.133 & $27(54.00)$ & $3(5.26)$ & $<0.001$ & $<0.001$ & 0.001 & 0.174 \\
\hline & \multirow{2}{*}{ TG } & $\mathrm{mg} / \mathrm{dL}$ & $\mathrm{Me}(\mathrm{IQR})$ & $132(88-182)$ & $101(76-138)$ & 0.008 & $183(156-225)$ & 97 (76-133) & $<0.001$ & $<0.001$ & $<0.001$ & 0.799 \\
\hline & & $\geq 150$ & $n(\%)$ & 41 (38.32) & 7 (18.42) & 0.025 & $38(76.00)$ & $3(5.26)$ & $<0.001$ & $<0.001$ & $<0.001$ & 0.088 \\
\hline
\end{tabular}


Table 1. Cont.

\begin{tabular}{|c|c|c|c|c|c|c|c|c|c|c|c|c|}
\hline Characteristics & Variable & $\mathrm{IU}$ & Parameter & $\begin{array}{c}\text { Ps Total } \\
(N=107)\end{array}$ & $\begin{array}{c}\text { HVs } \\
(N=38)\end{array}$ & $\begin{array}{c}p^{\mathrm{a}} \\
\text { Ps Total vs. } \\
\text { HVs }\end{array}$ & $\begin{array}{c}\text { Ps MetS (+) } \\
\quad(N=50)\end{array}$ & $\begin{array}{l}\text { Ps MetS }(-) \\
\quad(N=57)\end{array}$ & $\begin{array}{c}p^{\mathbf{b}} \\
\text { Ps MetS (+) } \\
\text { vs. Ps MetS } \\
\text { (-) vs. HVs }\end{array}$ & $\begin{array}{c}p^{\mathrm{a}} \\
\text { Ps MetS (+) } \\
\text { vs. Ps MetS } \\
\quad(-)\end{array}$ & $\begin{array}{c}p^{\mathrm{a}} \\
\text { Ps MetS (+) } \\
\text { vs. HVs }\end{array}$ & $\begin{array}{c}p^{\mathrm{a}} \\
\text { Ps MetS (-) } \\
\text { vs. HVs }\end{array}$ \\
\hline & \multirow{4}{*}{ CHOL/HDL } & ratio & $\mathrm{Me}(\mathrm{IQR})$ & $\begin{array}{c}3.54 \\
(2.79-4.89)\end{array}$ & $\begin{array}{c}3.16 \\
(2.50-4.08)\end{array}$ & 0.022 & $\begin{array}{c}4.72 \\
(3.52-6.03)\end{array}$ & $\begin{array}{c}3.19 \\
(2.47-3.64)\end{array}$ & $<0.001$ & $<0.001$ & $<0.001$ & 0.603 \\
\hline & & normal & $n(\%)$ & $63(58.88)$ & $26(68.42)$ & \multirow{3}{*}{0.004} & $15(30.00)$ & $48(84.21)$ & \multirow{3}{*}{$<0.001$} & \multirow{3}{*}{$<0.001$} & \multirow{3}{*}{$<0.001$} & \multirow{3}{*}{0.011} \\
\hline & & borderline & $n(\%)$ & $20(18.69)$ & $12(31.58)$ & & $14(28.00)$ & $6(10.53)$ & & & & \\
\hline & & high risk & $n(\%)$ & $24(22.43)$ & $0(0.00)$ & & $21(42.00)$ & $3(5.26)$ & & & & \\
\hline & \multirow{4}{*}{ LDL/HDL } & ratio & Me (IQR) & $\begin{array}{c}2.02 \\
(1.32-2.87)\end{array}$ & $\begin{array}{c}1.90 \\
(1.66-2.36)\end{array}$ & 0.414 & $\begin{array}{c}2.70 \\
(1.74-3.63)\end{array}$ & $\begin{array}{c}1.80 \\
(1.12-2.15)\end{array}$ & $<0.001$ & $<0.001$ & 0.001 & 0.132 \\
\hline & & normal & $n(\%)$ & $82(76.64)$ & $38(100.00)$ & \multirow{3}{*}{0.005} & $31(62.00)$ & $51(89.47)$ & \multirow{3}{*}{$<0.001$} & \multirow{3}{*}{0.002} & \multirow{3}{*}{$<0.001$} & \multirow{3}{*}{0.041} \\
\hline & & borderline & $n(\%)$ & $16(14.95)$ & $0(0.00)$ & & $11(22.00)$ & $5(8.77)$ & & & & \\
\hline & & high risk & $n(\%)$ & $9(8.41)$ & $0(0.00)$ & & $8(16.00)$ & $1(1.75)$ & & & & \\
\hline & \multirow{2}{*}{ TG/HDL } & ratio & Me (IQR) & $\begin{array}{c}2.63 \\
(1.63-4.60)\end{array}$ & $\begin{array}{c}2.02 \\
(1.42-2.91)\end{array}$ & 0.012 & $\begin{array}{c}4.70 \\
(2.98-6.02)\end{array}$ & $\begin{array}{c}1.80 \\
(1.19-2.58)\end{array}$ & $<0.001$ & $<0.001$ & $<0.001$ & 0.356 \\
\hline & & $\begin{array}{l}\text { above } \\
\text { normal }\end{array}$ & $n(\%)$ & $46(42.99)$ & $9(23.68)$ & 0.035 & $37(74.00)$ & 9 (15.79) & $<0.001$ & $<0.001$ & $<0.001$ & 0.487 \\
\hline \multirow{2}{*}{ Glucose } & \multicolumn{2}{|c|}{$\mathrm{mg} / \mathrm{dL}$} & $\mathrm{Me}$ (IQR) & 87 (82-95) & 87 (83-89) & 0.817 & 88 (82-97) & 87 (81-95) & 0.796 & 0.471 & 0.705 & 0.964 \\
\hline & \multicolumn{2}{|c|}{$\geq 100$} & $n(\%)$ & 15 (14.02) & $1(2.63)$ & 0.054 & $12(24.00)$ & $3(5.26)$ & 0.002 & 0.005 & 0.013 & 0.917 \\
\hline
\end{tabular}

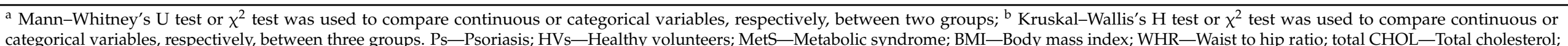
HDL-CHOL-High-density lipoprotein cholesterol; LDL-CHOL—Low-density lipoprotein cholesterol; TG—Triglycerides; Me—Median; IQR-Interquartile range. Significant differences are in bold. 
Table 2. Correlations between osteopontin serum concentration and characteristics of the psoriatic patients $(N=170)$.

\begin{tabular}{|c|c|c|c|c|c|}
\hline Characteristics & Variable & IU or Category & Test ${ }^{1}$ & Estimate & $p$ \\
\hline Age & & years & $\mathrm{r}$ & -0.158 & 0.056 \\
\hline \multirow{6}{*}{ Obesity } & \multirow{2}{*}{ BMI } & $\mathrm{kg} / \mathrm{m}^{2}$ & $\mathrm{r}$ & -0.010 & 0.916 \\
\hline & & overweight or obese vs. normal & $\mathrm{H}$ & 0.671 & 0.715 \\
\hline & \multirow{2}{*}{ WHR } & ratio & $\mathrm{r}$ & -0.118 & 0.227 \\
\hline & & $\geq 1$ vs. $<1$ & Z & 1.500 & 0.133 \\
\hline & \multirow{2}{*}{ Waist circumference } & $\mathrm{cm}$ & $\mathrm{r}$ & -0.105 & 0.280 \\
\hline & & $\geq 94 \mathrm{~cm}$ vs. $<94 \mathrm{~cm}$ & $\mathrm{Z}$ & 1.833 & 0.060 \\
\hline \multirow{4}{*}{ Blood pressure } & \multirow[b]{2}{*}{ Systolic } & $\mathrm{mm} \mathrm{Hg}$ & $\mathrm{r}$ & -0.070 & 0.477 \\
\hline & & $\geq 130$ vs. $<130$ & $\mathrm{Z}$ & 0.287 & 0.774 \\
\hline & \multirow{2}{*}{ Diastolic } & $\mathrm{mm} \mathrm{Hg}$ & $\mathrm{r}$ & -0.060 & 0.542 \\
\hline & & $\geq 85$ vs. $<85$ & Z & 0.347 & 0.729 \\
\hline \multirow{14}{*}{ Lipids } & \multirow{2}{*}{ Total CHOL } & $\mathrm{mg} / \mathrm{dL}$ & $\mathrm{r}$ & -0.274 & 0.004 \\
\hline & & $>190$ vs. $\leq 190$ & Z & 1.626 & 0.104 \\
\hline & \multirow{2}{*}{ LDL-CHOL } & $\mathrm{mg} / \mathrm{dL}$ & $\mathrm{r}$ & -0.114 & 0.244 \\
\hline & & $\geq 115$ vs. $<115$ & Z & 1.145 & 0.252 \\
\hline & \multirow{2}{*}{ HDL-CHOL } & $\mathrm{mg} / \mathrm{dL}$ & $\mathrm{r}$ & -0.058 & 0.562 \\
\hline & & $\leq 40$ vs. $>40$ & $\mathrm{Z}$ & 0.444 & 0.657 \\
\hline & \multirow{2}{*}{ TG } & $\mathrm{mg} / \mathrm{dL}$ & $\mathrm{r}$ & -0.250 & 0.009 \\
\hline & & $\geq 150$ vs. $<150$ & Z & 2.554 & 0.011 \\
\hline & \multirow{2}{*}{ CHOL/HDL } & - ratio & $\mathrm{r}$ & -0.094 & 0.334 \\
\hline & & borderline or high risk vs. normal & $\mathrm{H}$ & 1.904 & 0.386 \\
\hline & \multirow{2}{*}{ LDL/HDL } & ratio & $\mathrm{r}$ & -0.036 & 0.712 \\
\hline & & borderline or high risk vs. normal & $\mathrm{H}$ & 0.512 & 0.774 \\
\hline & \multirow{2}{*}{ TG/HDL } & ratio & $\mathrm{r}$ & -0.149 & 0.125 \\
\hline & & above normal vs. normal & $\mathrm{Z}$ & 1.988 & 0.047 \\
\hline \multirow{2}{*}{ Glucose } & & $\mathrm{mg} / \mathrm{dL}$ & $\mathrm{r}$ & -0.106 & 0.275 \\
\hline & & $\geq 100$ vs. $<100$ & $\mathrm{Z}$ & 0.542 & 0.588 \\
\hline
\end{tabular}

${ }^{1} \mathrm{r}$-Spearman's correlation coefficient, Z-Mann-Whitney's test, H-Kruskal-Wallis' test. BMI-Body mass index, WHR-Waist to hip ratio, total $\mathrm{CHOL}-$ Total cholesterol, HDL-CHOL-High-density lipoprotein cholesterol, LDL-CHOL-Low-density lipoprotein cholesterol, TG-Triglycerides. Significant differences are in bold.

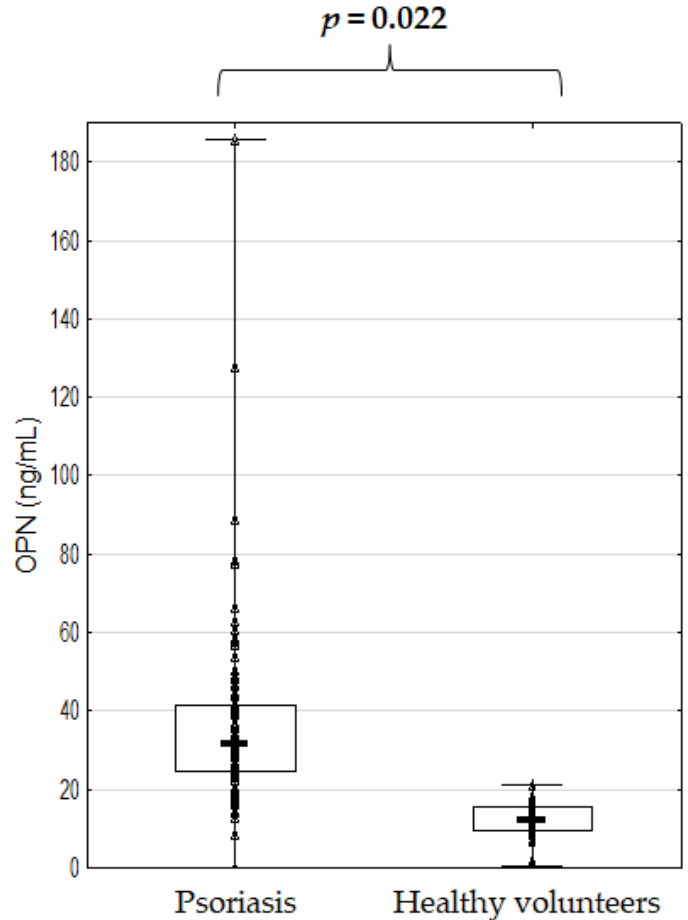

(a)

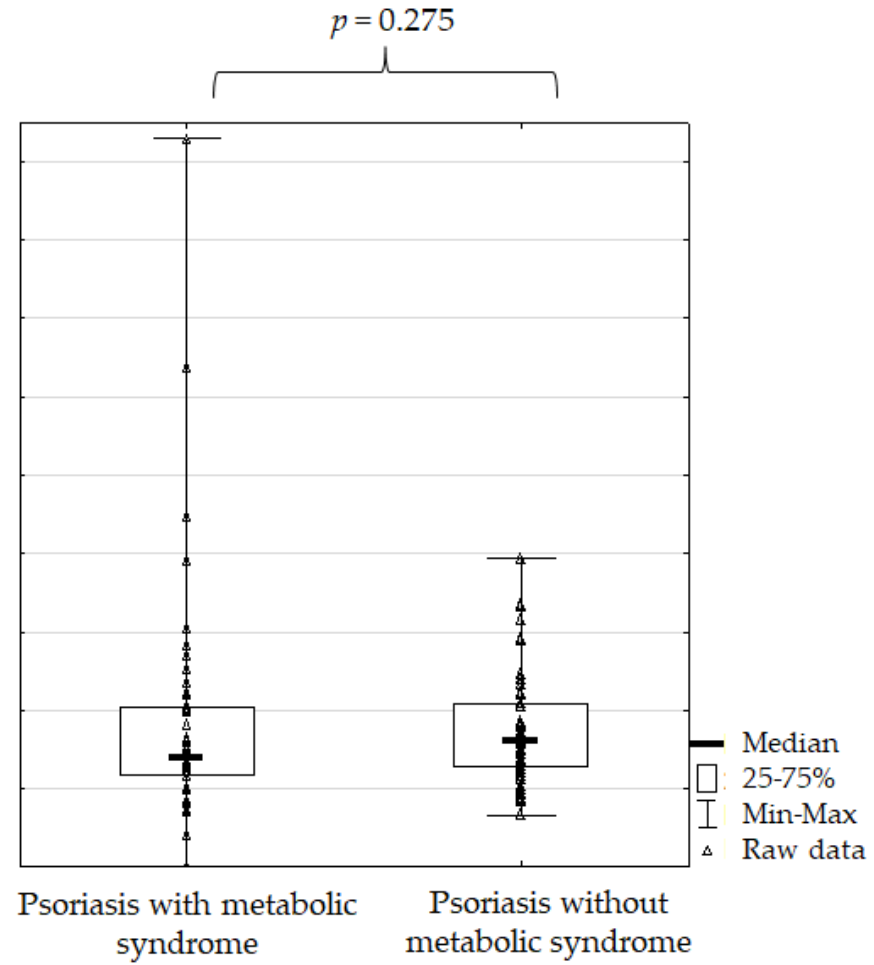

(b)

Figure 1. Osteopontin serum concentration (a) in the psoriatic patients versus healthy volunteers and (b) in the psoriatic patients with and without metabolic syndrome; $p$ for Mann-Whitney's U test; significant difference is in bold. 
However, OPN serum concentration in Ps patients correlated negatively with total CHOL $(r=-0.274, p=0.004)$ and TG $(r=-0.250, p=0.009)$. OPN serum concentration was significantly lower in Ps patients, with TG and TG/HDL ratio above standards in comparison to normal ranges (Figure 2).

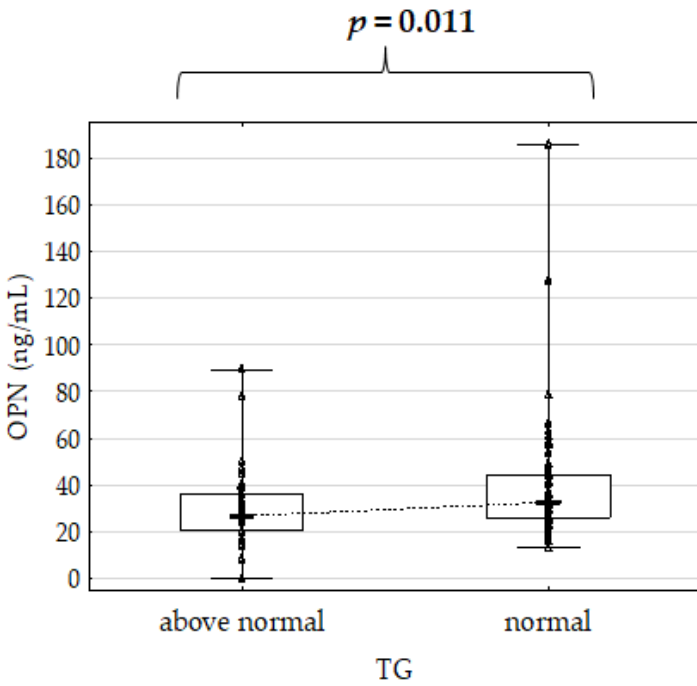

(a)

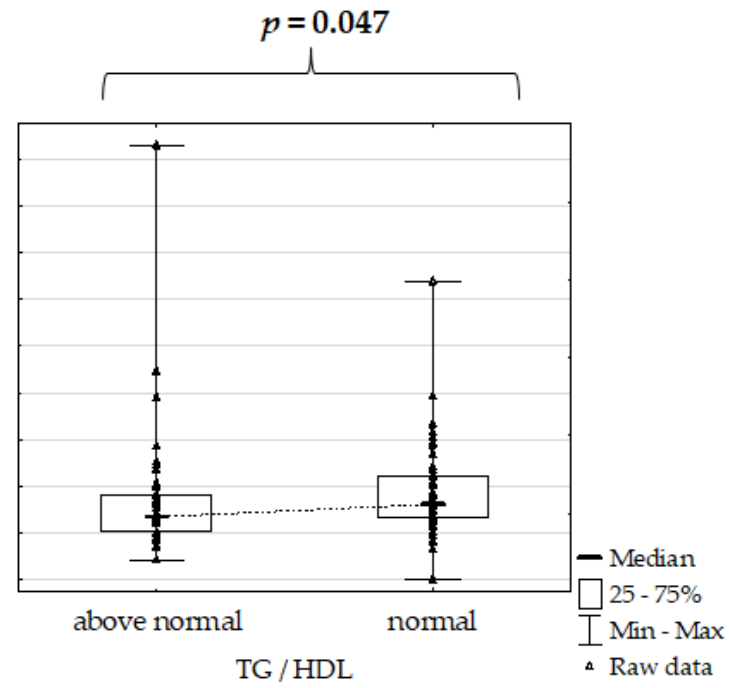

(b)

Figure 2. Osteopontin serum concentration (a) versus intervals of triglycerides (TG) and (b) triglycerides/high-density lipoprotein (TG/HDL) ratio in the psoriatic patients; $p$ for Mann-Whitney's U test; significant differences are in bold.

\subsection{OPN versus MetS Components in Ps Patients}

OPN serum concentration was significantly lower in Ps patients with hypertriglyceridemia in comparison to Ps patients without hypertriglyceridemia (Table 3). However, OPN serum concentration did not correlate to prevalence of abdominal obesity, low HDLCHOL, arterial hypertension and hyperglycemia in Ps patients (Table 3).

Table 3. Osteopontin serum concentration vs. prevalence of metabolic syndrome criteria in the psoriatic patients.

\begin{tabular}{|c|c|c|c|c|}
\hline \multirow[b]{2}{*}{ Criterion } & \multirow{2}{*}{$\begin{array}{c}n \\
\text { (\% of Psoriasis } \\
\text { Total } N=107 \text { ) }\end{array}$} & \multicolumn{2}{|c|}{ OPN Me (IQR) } & \multirow[b]{2}{*}{$p^{1}$} \\
\hline & & $\begin{array}{l}\text { Fulfilled Criterion of } \\
\text { Metabolic Syndrome }\end{array}$ & $\begin{array}{l}\text { Not Fulfilled Criterion } \\
\text { of Metabolic Syndrome }\end{array}$ & \\
\hline Abdominal obesity & $69(64.49)$ & $28.61(24.16-39.58)$ & $33.45(27.04-46.87)$ & 0.060 \\
\hline $\begin{array}{c}\text { Hypertriglyceridemia (TG } \geq 150 \text { or treatment } \\
\text { for dyslipidemia) }\end{array}$ & $45(42.06)$ & $27.04(23.28-36.38)$ & $33.07(25.97-44.85)$ & 0.012 \\
\hline $\begin{array}{l}\text { Low HDL-CHOL (HDL-CHOL } \leq 40 \text { or } \\
\text { treatment for dyslipidemia) }\end{array}$ & $36(33.64)$ & $31.54(24.59-42.81)$ & $31.65(24.39-41.01)$ & 0.690 \\
\hline $\begin{array}{c}\text { Arterial hypertension (systolic } \geq 130 \text { or } \\
\text { diastolic } \geq 85 \text { or treatment for hypertension) }\end{array}$ & $90(84.11)$ & $31.30(24.39-40.23)$ & $31.69(25.82-41.87)$ & 0.591 \\
\hline $\begin{array}{c}\text { Hyperglycemia (glucose } \geq 100 \text { or treatment } \\
\text { for type } 2 \text { diabetes) }\end{array}$ & $17(15.89)$ & $28.61(20.29-40.23)$ & $31.91(24.62-41.30)$ & 0.336 \\
\hline
\end{tabular}

\footnotetext{
1. Mann-Whitney's U test was used to compare OPN serum concentration between psoriatic patients with a fulfilled and not fulfilled criterion. OPN-Osteopontin, HDL-CHOL-High-density lipoprotein cholesterol, LDL-CHOL-Low-density lipoprotein cholesterol, TG-Triglycerides, Me-Median, IQR-Interquartile range. Significant differences are in bold.
}

\section{Discussion}

In the present study we aimed to assess the concentration of OPN regarding the presence of individual components of MetS. We observed no significant correlations be- 
tween OPN levels and obesity indices such as BMI $(p=0.916)$, WHR $(p=0.227)$ and waist circumference $(p=0.280)$ in the studied Ps patients.

Previously, a high level of OPN together with an increased amount of C-reactive protein (CRP) in atherosclerotic plaques was observed [22]. Both proteins may act synergistically in various inflammatory processes, including atherosclerosis; however, the exact mechanisms of interaction between CRP and OPN are still unknown. Yan et al. [23] demonstrated that plasma OPN concentration positively correlates with the severity of coronary artery disease in type 2 diabetes patients, which is independent of the occurrence of conventional cardiovascular risk factors.

In the course of Ps, increased triglycerides, LDL cholesterol and decreased HDL cholesterol values are the most frequently observed lipid disturbances [1,2]. In our study, the levels of total CHOL and TG as well as CHOL/HDL and TG/HDL ratios were significantly higher in Ps patients than in HVs, while LDL-CHOL did not differ between both groups. In the Ps patients, we observed a negative correlation between OPN levels and total CHOL as well as TG. OPN serum concentration did not correlate with LDL-CHOL and HDL-CHOL concentrations as well as CHOL/HDL and LDL/HDL ratios in the patients.

The OPN level was found to be significantly lower in Ps patients with MetS and hypertriglyceridemia than in Ps patients without MetS. Since 11 Ps patients with MetS $(22 \%)$ were treated with statins, this might explain the study results.

Similar to the results of our study, Kadry et al. [24] did not observe any correlation between plasma concentration of OPN and waist circumference, whereas Toossi et al. [25] did not find a correlation between the plasma concentration of OPN and BMI. Despite higher OPN levels in Ps patients, Duarte et al. [26] did not show any significant differences in OPN levels in obese Ps patients compared to those with normal body weight. Similar to our analyzed group, other studies concerning obese patients demonstrated elevated plasma OPN values [27-30]. However, no significant differences in OPN concentrations were reported when overweight and obese patients were compared [30].

Contrary to the present study, Robati et al. [31] observed a positive correlation between plasma OPN concentration and BMI in Ps patients.

Some studies indicated that OPN is involved in pathophysiological processes leading to the development of type 2 diabetes, especially when the disease co-occurred with atherosclerosis [13]. Yan et al. [23] found that the level of OPN is proportional to the severity of nephropathy and coronary heart disease in patients with type 2 diabetes. The authors suggested that OPN could be used as an indicator to evaluate the degree of diabetic vasculopathy [23]. Cai et al. [32] demonstrated that glucose can induce histone acetylation and methylation, which leads to upregulation of expression of OPN gene. Thus, by inhibiting histone methyltransferase, the deleterious effect of glucose can be reversed.

The study by Chen et al. [33] observed higher plasma OPN concentration in Ps patients with hypertension and diabetes. According to some authors, hypertension and chronic renal failure seem to be related to the level of OPN [13]. In patients with hypertension, Kurata et al. [22] found not only a positive correlation of OPN with CRP but also with aldosterone, which stimulates kidney fibroblasts secretion of OPN and collagen. Moreover, according to some reports, the concentration of OPN is associated not only with risk factors of CVDs, such as increased systolic and diastolic blood pressure, but also with higher BMI values and decreased HDL cholesterol [34,35].

Kadry et al. [24] also did not report a correlation between the value of OPN concentration in plasma and the presence of dyslipidemia. In the patients with hypercholesterolemia treated with atorvastatin, Tanaka et al. [36] observed significantly reduced plasma levels of OPN. The authors suggested that, among others, the beneficial effect of statins on CVDs may result from decreasing the OPN concentration in peripheral blood. In opposition to our study results, Robati et al. [31] demonstrated significant positive correlations between OPN concentration and total cholesterol as well as triglyceride levels in their Ps patients. The authors also found a positive correlation between levels of OPN in plasma and intima 
media wall thickness of the common carotid artery in the Ps patients compared to the control group.

It is suggested that the association between Ps and MetS may result from adipocyte dysfunction, chronic elevated levels of free fatty acids and increased levels of inflammatory cytokines (such as TNF- $\alpha$ and IL-6) which have a systemic effect on insulin regulation and lipid metabolism [37]. Such an association between Ps and MetS is a possible explanation of the mechanism of inflammatory march leading to the development of Ps co-morbidities [37]. Kadry et al. [24] found significantly higher values of plasma OPN concentration in Ps patients with MetS than in the control group. Another study by Kadry et al. [38] showed a positive correlation between the incidence of MetS and the PASI value. The authors believe that this may indicate common pathophysiological pathways of inflammation in both Ps and MetS. Abdel Hay et al. [39] showed significantly higher concentration of OPN in Ps patients compared to the control group as well as higher values in those patients who met the criteria of MetS. Interestingly, Yegin et al. [40] reported a positive correlation between OPN concentration and leptin, total CHOL, BMI and waist circumference and suggested that OPN may be a key mediator involved in the pathogenesis of MetS.

\section{Conclusions}

In conclusion, OPN as a marker of inflammation seems to be a significant protein involved in the development of Ps and MetS. However, studies to date do not allow for a precise definition of its role in these processes.

Author Contributions: Conceptualization, J.B. (Joanna Bartosińska), J.P.-K. and G.C.; methodology, D.R., J.B. (Joanna Bartosińska), J.P.-K. and G.C.; software, J.P.-K., D.R. and K.C.-S.; validation, J.B. (Joanna Bartosińska), J.P.-K. and G.C.; formal analysis, D.R. and J.B. (Jarosław Bartosiński); in-vestigation, M.K., J.P.-K. and G.C.; resources, J.P.-K.; data curation, J.P.-K. and M.K. and D.R.; writing—original draft preparation, J.P.-K., J.B. (Joanna Bartosińska) and B.S.-H.; writing—review and editing, G.C. and D.K.; visualization, J.B. (Jarosław Bartosiński) and J.K.; supervision, G.C.; project administration, J.B. (Jarosław Bartosinski) and J.K.; funding acquisition, J.P.-K. All authors have read and agreed to the published version of the manuscript.

Funding: Medical University of Lublin, Poland.

Institutional Review Board Statement: The study protocol com-plies with the ethical guidelines of the 1975 Declaration of Helsinki. The study was approved by the local ethics committee (KE0254/30/2016).

Informed Consent Statement: Informed consent was obtained from each individual.

Data Availability Statement: The data presented in this study are available on request in the Department of Dermatology, Venereology and Pediatric Dermatology, Medical University of Lublin (Poland). The data are not publicly available due to privacy restrictions.

Conflicts of Interest: The authors declare no conflict of interest.

$\begin{array}{ll}\text { Abbreviations } \\ \text { Ps } & \text { Psoriasis } \\ \text { MetS } & \text { Metabolic syndrome } \\ \text { OPN } & \text { Osteopontin } \\ \text { HVs } & \text { Healthy volunteers } \\ \text { CVDs } & \text { Cardiovascular diseases } \\ \text { TNF- } \alpha & \text { Tumor necrosis factor } \alpha \\ \text { IL } & \text { Interleukin } \\ \text { ELISA } & \text { Enzyme-linked immunosorbent assay } \\ \text { CRP } & \text { C-reactive protein } \\ \text { CHOL } & \text { Cholesterol }\end{array}$




$\begin{array}{ll}\text { TG } & \text { Triglycerides } \\ \text { HDL-CHOL } & \text { High-density lipoprotein cholesterol } \\ \text { LDL-CHOL } & \text { Low-density lipoprotein cholesterol } \\ \text { VLDL } & \text { Very-low-density lipoprotein } \\ \text { BMI } & \text { Body mass index } \\ \text { WHR } & \text { Waist-to-hip ratio }\end{array}$

\section{References}

1. Gisondi, P.; Bellinato, F.; Girolomoni, G.; Albanesi, C. Pathogenesis of Chronic Plaque Psoriasis and Its Intersection with Cardio-Metabolic Comorbidities. Front. Pharmacol. 2020, 11, 117. [CrossRef]

2. Egeberg, A.; Gisondi, P.; Carrascosa, J.M.; Warren, R.B.; Mrowietz, U. The role of the interleukin-23/Th17 pathway in cardiometabolic comorbidity associated with psoriasis. J. Eur. Acad. Dermatol. Venereol. 2020, 34, 1695-1706. [CrossRef]

3. Furue, M.; Tsuji, G.; Chiba, T.; Kadono, T. Cardiovascular and Metabolic Diseases Comorbid with Psoriasis: Beyond the Skin. Intern. Med. 2017, 56, 1613-1619. [CrossRef] [PubMed]

4. Lønnberg, A.S.; Skov, L. Co-morbidity in psoriasis: Mechanisms and implications for treatment. Expert. Rev. Clin. Immunol. 2017, 13, 27-34. [CrossRef] [PubMed]

5. Owczarczyk-Saczonek, A.; Placek, W. Interleukin-17 as a factor linking the pathogenesis of psoriasis with metabolic disorders. Int. J. Dermatol. 2017, 56, 260-268. [CrossRef] [PubMed]

6. $\quad$ Egeberg, A.; Skov, L.; Joshi, A.A.; Mallbris, L.; Gislason, G.H.; Wu, J.J.; Rodante, J.; Lerman, J.B.; Ahlman, M.A.; Gelfand, J.M.; et al. The relationship between duration of psoriasis, vascular inflammation, and cardiovascular events. J. Am. Acad. Dermatol. 2017, 77, 650-656. [CrossRef] [PubMed]

7. Armstrong, E.J.; Harskamp, C.T.; Armstrong, A.W. Psoriasis and major adverse cardiovascular events: A systematic review and meta-analysis of observational studies. J. Am. Heart Assoc. 2013, 2, e000062. [CrossRef]

8. Kothari, A.N.; Arffa, M.L.; Chang, V.; Blackwell, R.H.; Syn, W.K.; Zhang, J.; Mi, Z.; Kuo, P.C. Osteopontin-A Master Regulator of Epithelial-Mesenchymal Transition. J. Clin. Med. 2016, 5, 39. [CrossRef]

9. Clemente, N.; Raineri, D.; Cappellano, G.; Boggio, E.; Favero, F.; Soluri, M.F.; Dianzani, C.; Comi, C.; Dianzani, U.; Chiocchetti, A. Osteopontin Bridging Innate and Adaptive Immunity in Autoimmune Diseases. J. Immunol. Res. 2016, 13, 1-15. [CrossRef]

10. Gürsoy, G.; Acar, S.; Alagöz, S. Osteopontin: A multifunctional molecule. J. Med. Med. Sci. 2010, 1, 55-60.

11. Scatena, M.; Liaw, L.; Giachelli, C.M. Osteopontin: A multifunctional molecule regulating chronic inflammation and vascular disease. Arterioscler. Thromb. Vasc. Biol. 2007, 27, 2302-2309. [CrossRef] [PubMed]

12. Subramani, V.; Thiyagarajan, M.; Malathi, N.; Rajan, S.T. OPN -Revisited. J. Clin. Diagn. Res. 2015, 9, ZE10-ZE13. [CrossRef]

13. Wolak, T. Osteopontin-A multi-modal marker and mediator in atherosclerotic vascular disease. Atherosclerosis 2014, $236,327-337$. [CrossRef] [PubMed]

14. Cho, H.J.; Cho, H.J.; Kim, H.S. Osteopontin: A multifunctional protein at the crossroads of inflammation, atherosclerosis, and vascular calcification. Curr. Atheroscler. Rep. 2009, 11, 206-213. [CrossRef]

15. Sodek, J.; Batista Da Silva, A.P.; Zohar, R. Osteopontin and mucosal protection. J. Dent. Res. 2006, 85, 404-415. [CrossRef]

16. Ding, Y.; Chen, J.; Cui, G.; Wei, Y.; Lu, C.; Wang, L.; Diao, H. Pathophysiological role of osteopontin and angiotensin II in atherosclerosis. Biochem. Biophys. Res. Commun. 2016, 471, 5-9. [CrossRef] [PubMed]

17. Kahles, F.; Findeisen, H.M.; Bruemmer, D. Osteopontin: A novel regulator at the cross roads of inflammation, obesity and diabetes. Mol. Metab. 2014, 3, 384-393. [CrossRef]

18. Zhang, Y.M.; Li, M.X.; Tang, Z.; Wang, C.H. Wogonin suppresses osteopontin expression in adipocytes by activating PPAR $\alpha$. Acta Pharmacol. Sin. 2015, 36, 987-997. [CrossRef]

19. Hoffman, E.P.; Gordish-Dressman, H.; McLane, V.D.; Devaney, J.M.; Thompson, P.D.; Visich, P.; Gordon, P.M.; Pescatello, L.S.; Zoeller, R.F.; Moyna, N.M.; et al. Alterations in osteopontin modify muscle size in females in both humans and mice. Med. Sci. Sports Exerc. 2013, 45, 1060-1068. [CrossRef]

20. Cho, E.H.; Cho, K.H.; Lee, H.A.; Kim, S.W. High serum osteopontin levels are associated with low bone mineral density in postmenopausal women. J. Korean Med. Sci. 2013, 28, 1496-1499. [CrossRef]

21. Fodor, D.; Bondor, C.; Albu, A.; Simon, S.P.; Craciun, A.; Muntean, L. The value of osteopontin in the assessment of bone mineral density status in postmenopausal women. J. Investig. Med. 2013, 61, 15-21. [CrossRef]

22. Kurata, M.; Okura, T.; Irita, J.; Enomoto, D.; Nagao, T.; Jotoku, M.; Miyoshi, K.; Higaki, J. The relationship between osteopontin and adiponectin in patients with essential hypertension. Clin. Exp. Hypertens. 2010, 32, 358-363. [CrossRef] [PubMed]

23. Yan, X.; Sano, M.; Lu, L.; Wang, W.; Zhang, Q.; Zhang, R.; Wang, L.; Chen, Q.; Fukuda, K.; Shen, W. Plasma concentrations of osteopontin, but not thrombin-cleaved osteopontin, are associated with the presence and severity of nephropathy and coronary artery disease in patients with type 2 diabetes mellitus. Cardiovasc. Diabetol. 2010, 9, 70. [CrossRef] [PubMed]

24. Kadry, D.; Hegazy, R.A.; Rashed, L. Osteopontin and adiponectin: How far are they related in the complexity of psoriasis? Arch Dermatol. Res. 2013, 305, 939-944. [CrossRef] [PubMed]

25. Toossi, P.; Sadat Amini, S.H.; Sadat Amini, M.S.; Partovi Kia, M.; Enamzade, R.; Kazeminejad, A.; Esmaeily Radvar, S.; Younespour, S. Assessment of serum levels of osteopontin, selenium and prolactin in patients with psoriasis compared with healthy controls, and their association with psoriasis severity. Clin. Exp. Dermatol. 2015, 40, 741-746. [CrossRef] 
26. Duarte, G.V.; Boeira, V.; Correia, T.; Porto-Silva, L.; Cardoso, T.; Macedo, M.N.; Oliveira, M.F.; Carvalho, E. Osteopontin, CCL5 and CXCL9 are independently associated with psoriasis, regardless of the presence of obesity. Cytokine 2015, 74, 287-292. [CrossRef]

27. Komorowski, J.; Jankiewicz-Wika, J.; Kolomecki, K.; Cywinski, J.; Piestrzeniewicz, K.; Swiętoslawski, J.; Stepien, H. Systemic blood osteopontin, endostatin, and E-selectin concentrations after vertical banding surgery in severely obese adults. Cytokine 2011, 55, 56-61. [CrossRef]

28. Ahmad, R.; Al-Mass, A.; Al-Ghawas, D.; Shareif, N.; Zghoul, N.; Melhem, M.; Hasan, A.; Al-Ghimlas, F.; Dermime, S.; Behbehani, K. Interaction of osteopontin with IL-18 in obese individuals: Implications for insulin resistance. PLoS ONE 2013, 8, e63944. [CrossRef]

29. Buback, F.; Renkl, A.C.; Schulz, G.; Weiss, J.M. Osteopontin and the skin: Multiple emerging roles in cutaneous biology and pathology. Exp. Dermatol. 2009, 18, 750-759. [CrossRef]

30. Gómez-Ambrosi, J.; Catalán, V.; Ramírez, B.; Rodríguez, A.; Colina, I.; Silva, C.; Rotellar, F.; Muguet, C.; Gil, M.J.; Cienfuegos, J.A.; et al. Plasma osteopontin levels and expression in adipose tissue are increased in obesity. J. Clin. Endocrinol. Metab. 2007, 92, 3719-3727. [CrossRef]

31. Robati, R.M.; Partovi-Kia, M.; Sadat-Amini, H.; Haghighatkhah, H.R.; Younespour, S.; Toossi, P. Serum osteopontin level and common carotid artery intima-media wall thickness in psoriasis. Int. J. Dermatol. 2016, 55, 262-267. [CrossRef]

32. Cai, M.; Bompada, P.; Atac, D.; Laakso, M.; Groop, L.; De Marinis, Y. Epigenetic regulation of glucose-stimulated osteopontin (OPN) expression in diabetic kidney. Biochem. Biophys. Res. Commun. 2016, 469, 108-113. [CrossRef]

33. Chen, Y.J.; Shen, J.L.; Wu, C.Y.; Chang, Y.T.; Chen, C.M.; Lee, F.Y. Elevated plasma osteopontin level is associated with occurrence of psoriasis and is an unfavorable cardiovascular risk factor in patients with psoriasis. J. Am. Acad. Dermatol. 2009, 60, 225-230. [CrossRef]

34. Irita, J.; Okura, T.; Kurata, M.; Miyoshi, K.; Fukuoka, T.; Higaki, J. Osteopontin in rat renal fibroblasts: Functional properties and transcriptional regulation by aldosterone. Hypertension 2008, 51, 507-513. [CrossRef]

35. Barchetta, I.; Alessandri, C.; Bertoccini, L.; Cimini, F.A.; Taverniti, L.; Di Franco, M.; Fraioli, A.; Baroni, M.G.; Cavallo, M.G. Increased circulating osteopontin levels in adult patients with type 1 diabetes mellitus and association with dysmetabolic profile. Eur. J. Endocrinol. 2016, 174, 187-192. [CrossRef] [PubMed]

36. Tanaka, N.; Momiyama, Y.; Ohmori, R.; Yonemura, A.; Ayaori, M.; Ogura, M.; Sawada, S.; Kusuhara, M.; Nakamura, H.; Ohsuzu, F. Effect of atorvastation on plasma osteopontin levels in patients with hypercholesterolemia. Arterioscler. Thromb. Vasc. Biol. 2006, 26, 129-130. [CrossRef] [PubMed]

37. Krueger, J.G.; Brunner, P.M. Interleukin-17 alters the biology of many cell types involved in the genesis of psoriasis, systemic inflammation and associated comorbidities. Exp. Dermatol. 2018, 27, 115-123. [CrossRef]

38. Kadry, D.; Rashed, R. Plasma and tissue osteopontin in relation to plasma selenium in patients with psoriasis. J. Eur. Acad. Dermatol. Venereol. 2012, 26, 66-70. [CrossRef] [PubMed]

39. Abdel Hay, R.; Nour-Edin, F.; Hegazy, R.; Khadiga, S.; Rashed, L. Expression of osteopontin genotypes (T-4754-C and A-9138-C) in psoriasis and their relation to metabolic syndrome. J. Dermatol. Sci. 2014, 75, 150-153. [CrossRef]

40. Yegin, Z.A.; İyidir, Ö.T.; Demirtaş, C.; Suyanı, E.; Yetkin, İ.; Paşaoğlu, H.; İlhan, Ç.; Sucak, G.T. The interplay among iron metabolism, endothelium and inflammatory cascade in dysmetabolic disorders. J. Endocrinol. Invest. 2015, 38, 333-338. [CrossRef] 